TRANSACTIONS OF THE

AMERICAN MATHEMATICAL SOCIETY

Volume 365, Number 3, March 2013, Pages 1551-1575

S 0002-9947(2012)05692-3

Article electronically published on October 1, 2012

\title{
THE CALABI-YAU EQUATION ON 4-MANIFOLDS OVER 2-TORI
}

\author{
A. FINO, Y.Y. LI, S. SALAMON, AND L. VEZZONI
}

\begin{abstract}
This paper pursues the study of the Calabi-Yau equation on certain symplectic non-Kähler 4-manifolds, building on a key example of Tosatti and Weinkove in which more general theory had proved less effective. Symplectic 4-manifolds admitting a 2-torus fibration over a 2-torus base $\mathbb{T}^{2}$ are modelled on one of three solvable Lie groups. Having assigned an invariant almost-Kähler structure and a volume form that effectively varies only on $\mathbb{T}^{2}$, one seeks a symplectic form with this volume. Our approach simplifies the previous analysis of the problem and establishes the existence of solutions in various other cases.
\end{abstract}

\section{INTRODUCTION}

Let $(M, J, \Omega)$ be a $2 n$-dimensional compact Kähler manifold with associated complex structure $J$ and symplectic form $\Omega$. Yau's theorem implies that any representative of the first Chern class of $M$ can be written as the Ricci curvature of a unique Kähler metric whose 2-form $\tilde{\Omega}$ lies in the same cohomology class of $\Omega$ [28]. This can be restated by saying that for every volume form $\sigma$ on $M$ satisfying

$$
\int_{M} \sigma=\int_{M} \Omega^{n}
$$

there exists a unique Kähler form $\tilde{\Omega}$ such that $[\tilde{\Omega}]=[\Omega]$ and

$$
\tilde{\Omega}^{n}=\sigma .
$$

The same problem can be posed in an 'almost-Kähler' context, when $\Omega$ remains closed but $J$ is merely an almost-complex structure. The latter is still orthogonal relative to a Riemannian metric $g$ for which $\Omega(X, Y)=g(J X, Y)$, and

$$
\tilde{\Omega}=\Omega+d \alpha
$$

is again assumed to be a positive-definite $(1,1)$-form relative to $J$. In this set-up, (1.1), (1.2), (1.3) constitute the Calabi-Yau problem. Donaldson proved in [5] that its solution is unique in four dimensions (see Proposition 3.2), and we provide a dimensionally reduced version of this proof in Section 4. In the almost-Kähler and Hermitian cases, the Calabi-Yau problem has been further studied in extensive papers by Tosatti, Weinkove, Yau [23, 24] and others.

A sufficient condition for the existence of solutions to the Calabi-Yau equation in terms of the Chern connection was given in [23], but this condition fails in the strictly almost-Kähler case when a scalar curvature function is negative (Proposition 3.3). The simplest such case is that of a Kodaira surface. The latter is a

Received by the editors April 11, 2011 and, in revised form, August 6, 2011.

2010 Mathematics Subject Classification. Primary 53C25, 35J60, 53 D35. 
discrete quotient of $\mathbb{C}^{2}$ and carries a holomorphic symplectic form trivializing the canonical bundle [15. It thereby becomes a symplectic non-Kähler manifold that is realized as a principal $T^{2}$-bundle over a 2 -torus base $\mathbb{T}^{2}$. (Throughout this paper, we use blackboard font for the base, to avoid confusion.) As such, it is often called the Kodaira-Thurston manifold [22] and is a valuable vehicle for testing new phenomena in differential geometry [1, 8].

In [25, Tosatti and Weinkove solved the Calabi-Yau problem on the KodairaThurston manifold $M$ for every $T^{2}$-invariant volume form $\sigma$. The purpose of this paper is threefold:

(i) To extend the analysis of [25] to emphasize invariance of the solution by the $S^{1}$ action that rotates the symplectic forms $\Omega_{2}, \Omega_{3}$ and associated almost-complex structures $J_{2}, J_{3}$.

(ii) To generalize the theory so as to tackle other $T^{2}$-bundles over $\mathbb{T}^{2}$ and assigned volume forms from the base.

(iii) To set up the problem with arbitrary invariant metrics, as a first step to tackling more general initial data.

We accomplish (i) with the aid of Theorem 4.4, reducing the problem to a standard Monge-Ampère equation on the base, which can then be solved by an established theorem of the second author [16]. This is based on the existence of a potential for the holomorphic symplectic structure, which is not surprising given that the relevant fibration is analogous to a moment mapping. The approach makes the solutions (or at least, the method of solution) more explicit, and this is our aim in the other cases.

By work of Ue 26, any orientable 2-torus bundle $M$ over a 2-torus is a smooth quotient $\Gamma \backslash X$ covered by solvmanifold. In practice, there are just three candidates for $X$ that we need consider in (ii), namely $N i l^{3} \times \mathbb{R}, N i l^{4}$ and $S o l^{3} \times \mathbb{R}$, all diffeomorphic to $\mathbb{R}^{4}$, in which $N i l^{3}, N i l^{4}$ are nilpotent Lie groups and $S o l^{3}$ is a particular solvable Lie group. It is well known (and we shall demonstrate) that such quotients all admit symplectic structures. The notion of invariant almost-Kähler structure makes sense in this context, meaning one induced from a left-invariant structure on $X$ which is invariant by $\Gamma$. As regards (iii), we make most progress in the nilpotent case:

Theorem 1.1. Let $M=\Gamma \backslash X$ with $X=N i l^{3} \times \mathbb{R}$ or $N i l^{4}$, and suppose that $M$ admits an invariant almost-Kähler structure $(g, J, \Omega)$, and a $T^{2}$-fibration $\pi: M \rightarrow$ $\mathbb{T}^{2}$ whose fibres are Lagrangian. Then for every normalized volume form $\sigma=\mathrm{e}^{F} \Omega^{2}$ with $F \in C^{\infty}\left(\mathbb{T}^{2}\right)$, the corresponding Calabi-Yau problem has a unique solution.

The Lagrangian condition may or may not apply in the 2-step case, but is automatic when $M$ is modelled on $N i l^{4}$. The latter case leads to a generalized Monge-Ampère equation on the 2-torus base, for which we establish solutions in Section 6. The same equation is needed to establish Theorem 1.1 for a second fibration $\Gamma \backslash\left(N i l^{3} \times \mathbb{R}\right) \rightarrow \mathbb{T}^{2}$ in which the Lagrangian condition is not automatic, and in Section 7. we exhibit this 2-step situation as a limiting case of the 3 -step one.

Compact quotients of $\mathrm{Sol}^{3} \times \mathbb{R}$ do not admit Lagrangian fibrations, and therefore Theorem 1.1 does not apply. On the other hand, if $\Omega$ is a symplectic form on the total space of any $T^{2}$-fibration $M^{4} \rightarrow \mathbb{T}^{2}$ for which the fibres are not Lagrangian, 
then we can choose a compatible almost-complex structure $J$ such that $\pi$ is $J$ holomorphic. An elementary argument (Proposition 3.1) shows that the CalabiYau problem for $(g, J, \Omega)$ also has a solution in this very special case. We extend this argument in Section 8 by considering a foliation of $M=\Gamma \backslash\left(\mathrm{Sol}^{3} \times \mathbb{R}\right)$ by 2dimensional leaves transverse to $\pi$ that are holomorphic relative to any invariant almost-Kähler structure.

The second author was partially supported by NSF grant DMS-0701545 and the Program for Changjiang Scholars and Innovative Research Team in University in China. The other authors were funded by the Italian MIUR project Differential Geometry and Global Analysis (PRIN07).

\section{A BRief Classification of $T^{2}$-BundLes Over $\mathbb{T}^{2}$}

Orientable bundles with a 2-torus fibre $T^{2}$ over a 2 -torus base $\mathbb{T}^{2}$ were classified by Sakamoto and Fukuhara in [19], and it was shown by Ue that all these manifolds are geometric. By a geometric 4-manifold in the sense of Thurston one means a pair $(X, G)$ where $X$ is a complete, simply connected Riemannian 4-manifold, and $G$ is a group of isometries acting transitively on $X$ that contains a discrete subgroup $\Gamma$ such that $\Gamma \backslash X$ has finite volume. Since the stabilizer of $G$ at a point in $X$ is compact, so $\Gamma \backslash X$ is compact if and only if $\Gamma \backslash G$ is compact.

Two pairs $(X, G)$ and $\left(X^{\prime}, G^{\prime}\right)$ define the same geometry if the actions of $G$ and $G^{\prime}$ are related by a diffeomorphism $f: X \rightarrow X^{\prime}$. There are 19 4-dimensional geometries $X$ which have been classified by Filipkiewicz [7, 11]. In this paper we will only consider the ones which are orientable $T^{2}$-bundles over $\mathbb{T}^{2}$, and we will take $G$ to be the identity component of the isometry group of $X$. The relevant pairs $(X, G)$ are as follows.

(0) $X=E^{4}$ (Euclidean 4-space) and $G=S O(4) \ltimes \mathbb{R}^{4}$, the semidirect product of translations and rotations.

(1) $X=N i l^{3} \times E^{1}$, where $N i l^{3}$ is the 3 -dimensional Heisenberg group of matrices

$$
H_{x, y, z}=\left(\begin{array}{ccc}
1 & x & z \\
0 & 1 & y \\
0 & 0 & 1
\end{array}\right)
$$

under multiplication. Here, $G$ is spanned by $\mathrm{Nil}^{3}$ acting on itself on the left, by translation in the $E^{1}$-factor and by an additional isometric action of $S^{1}$ :

$$
\begin{aligned}
(x, y, z) \mapsto & (x \cos \theta+y \sin \theta,-x \sin \theta+y \cos \theta, z \\
& \left.+\frac{1}{2} \sin \theta\left(y^{2} \cos \theta-x^{2} \cos \theta-2 x y \sin \theta\right)\right) .
\end{aligned}
$$

(2) $X=N i l^{4}=\mathbb{R} \ltimes \mathbb{R}^{3}$ is the group of real matrices

$$
\left(\begin{array}{cccc}
1 & t & \frac{1}{2} t^{2} & x \\
0 & 1 & t & y \\
0 & 0 & 1 & z \\
0 & 0 & 0 & 1
\end{array}\right)
$$


under multiplication. Given that $N i l^{4}$ coincides with the identity component of its isometry group, $G=X$ acts on itself on the left.

(3) $X=S_{\text {Sol }}^{3} \times E^{1}$, where $S_{0 l}^{3}=\mathbb{R} \ltimes_{\varphi} \mathbb{R}^{2}$ is a solvable Lie group with $\varphi(t)=$ $\left(\begin{array}{cc}\mathrm{e}^{t} & 0 \\ 0 & \mathrm{e}^{-t}\end{array}\right)$, and $G=X$ acts by left multiplication:

$$
\left(x_{0}, y_{0}, z_{0}, t_{0}\right)(x, y, z, t)=\left(x_{0}+\mathrm{e}^{t_{0}} x, y_{0}+\mathrm{e}^{-t_{0}} y, z_{0}+z, t_{0}+t\right) .
$$

By a suitable choice of basis, we can specify the Lie algebras of the three nonabelian goups as follows:

$$
\begin{aligned}
N i l^{3} \times \mathbb{R} & \longleftrightarrow(0,0,0,12), \\
N i l^{4} & \longleftrightarrow(0,13,0,12), \\
S_{o l} l^{3} \times \mathbb{R} & \longleftrightarrow(0,0,13,41) .
\end{aligned}
$$

The digits on the right encode the exterior derivative relative to an invariant coframe $\left(e^{1}, \ldots, e^{4}\right)$, and in any case we shall use the notation $e^{12 \cdots}=e^{1} \wedge e^{2} \wedge \cdots$ for simple differential forms. For example, in case (2), we opt to define $e^{1}=-d t, e^{2}=d y-t d z$, $e^{3}=d z$, and (see (5.2) ) we obtain

$$
d e^{i}= \begin{cases}0 & i=1,3 \\ e^{13}, & i=2 \\ e^{12}, & i=4 .\end{cases}
$$

If one rescales so that the middle line becomes $d e^{2}=\lambda e^{13}$, case (1) is the result of letting $\lambda$ tend to 0 . This observation is important for the sequel.

Note that $\mathrm{Sol}^{3}$ refers to just one particular solvable Lie group, which is distinct, for example, from that with a Lie group corresponding to $(0,13,14)$ that has no cocompact lattice.

The diffeomorphism classes of (the total space of) $T^{2}$-bundles over $\mathbb{T}^{2}$ can be summarized in Geiges' eight families [9, Table 1]. By [9, Theorem 3], the total space is Kähler if and only the geometric type is $E^{4}$; this happens for two families, which are not interesting from our point of view. For each of the other six families we next describe $X, \Gamma$, the monodromy matrices $A_{1}, A_{2} \in S L(2, \mathbb{Z})$ along the curves $\gamma_{1}, \gamma_{2}$ generating $\pi_{1}\left(\mathbb{T}^{2}\right)$, as well as the Euler class $(m, n)$ for the corresponding $T^{2}$-bundle, which can be then described by the generators of $\Gamma$. By [27] for some lifts $\tilde{\gamma}_{i}$ to $M$ we have

$$
\pi_{1}(M)=\left\langle\tilde{\gamma}_{1}, \tilde{\gamma}_{2}, l, h \mid[l, h]=1, \tilde{\gamma}_{i}(l, h) \gamma_{i}^{-1}=(l, h) A_{i}, \quad\left[\tilde{\gamma}_{1}, \tilde{\gamma}_{2}\right]=l^{m} h^{n}\right\rangle .
$$

The discrete group $\Gamma$ arises as the image of a faithful representation $\rho$ from $\pi_{1}(M)$ to the group of orientation-preserving isometries of $X$, so $\Gamma$ is generated by $\rho\left(\tilde{\gamma}_{1}\right)$, $\rho\left(\tilde{\gamma}_{2}\right), \rho(l), \rho(h)$. To simplify the notation, we identify a generating map with the image of $(x, y, z, t)$ under this map. Unless otherwise stated, there is no restriction on $(m, n)$. Following [9] and [27, we have the six families:

(1 $\left.1_{\mathrm{a}}\right) \quad X=N i l^{3} \times E^{1}$, with $\Gamma$ generated by

$$
(x+1, y, z+\lambda y, t),(x, y+1, z, t),(x, y, z+1, t),(x, y, z, t+1) .
$$

Here $A_{1}=A_{2}=I$ (the identity matrix), $\lambda \neq 0$ and $(m, n) \neq(0,0)$. 
(1 $\left.1_{\mathrm{b}}\right) \quad X=N i l^{3} \times E^{1}$, with $\Gamma$ generated by

$$
\begin{aligned}
& \left(x, y, z+\beta_{1}, t\right),\left(x+\alpha_{1}, y, z+\alpha_{1} y, t\right), \\
& \left(x+\alpha_{2}, y, z+\alpha_{2} y+\beta_{2}, t+1\right),\left(-x, y+1,-z+\gamma_{3}, t\right) .
\end{aligned}
$$

Here $A_{1}=\left(\begin{array}{cc}-1 & \lambda \\ 0 & -1\end{array}\right), A_{2}=I$, with $\beta_{1}, \alpha_{1}, \lambda$ all non-zero.

(1 $) \quad X=N i l^{3} \times E^{1}$, with $\Gamma$ generated by

$$
\begin{aligned}
& \left(x, y, z+\beta_{1}, t\right),\left(x+\alpha_{1}, y, z+\alpha_{1} y, t\right), \\
& \left(x+\alpha_{3}, y+1, z+\alpha_{3} y+\beta_{4}, t\right),(-x, y,-z, t+1) .
\end{aligned}
$$

Here $A_{1}=\left(\begin{array}{cc}1 & \lambda \\ 0 & 1\end{array}\right), A_{2}=-I$, with $\beta_{1}, \alpha_{1}, \lambda$ all non-zero.

(2) $X=N i l^{4}$, with $\Gamma$ generated by

$$
\begin{aligned}
& \left(x+\alpha_{1}, y, z, t\right), \quad\left(x, y+\beta_{1}, z, t\right), \\
& \left(x+y+\frac{1}{2} z+\alpha_{2}, y, z, t+1\right), \quad\left(x+\alpha_{3} y+\beta_{3}, z+1, t\right) .
\end{aligned}
$$

Here $A_{1}=\left(\begin{array}{cc}1 & \lambda \\ 0 & 1\end{array}\right), A_{2}=I$, with $\beta_{1}=\frac{1}{n}, \lambda=\frac{1}{n \alpha_{1}}, \beta_{3}=\frac{m}{\alpha_{1}}-\frac{1}{2}$ and $n \neq 0$.

(3a) $X=S_{\mathrm{a}} \mathrm{l}^{3} \times E^{1}$, with $\Gamma$ generated by

$$
\begin{array}{ll}
\left(x+\alpha_{1}, y+\beta_{1}, z, t\right), & \left(x+\alpha_{2}, y+\beta_{2}, z, t\right), \\
\left(x+\alpha_{3}, y+\beta_{3}, z+1, t\right), & \left(\epsilon \mathrm{e}^{\delta} x+\alpha_{4}, \epsilon \mathrm{e}^{-\delta} y+\beta_{4}, z, t+\delta\right) .
\end{array}
$$

Here $A_{1} \in S L(2, \mathbb{Z}), A_{2}=I$, and $\epsilon= \pm 1$.

(3b) $X=S_{\mathrm{b}} \mathrm{l}^{3} \times E^{1}$, with $\Gamma$ generated by

$$
\begin{aligned}
& \left(x+\alpha_{1}, y+\beta_{1}, z, t\right), \quad\left(x+\alpha_{2}, y+\beta_{2}, z, t\right), \\
& \left(-x+\alpha_{3},-y+\beta_{3}, z+1, t\right), \quad\left(\epsilon \mathrm{e}^{\delta} x+\alpha_{4}, \epsilon \mathrm{e}^{-\delta} y+\beta_{4}, z, t+\delta\right) .
\end{aligned}
$$

Here $A_{1} \in S L(2, \mathbb{Z}), A_{2}=-I$, and $\epsilon=1$. As in $\left(3_{\mathrm{a}}\right)$, there are various relations (which we omit) between the other parameters.

The total space $M$ of an orientable $T^{2}$-bundle over $\mathbb{T}^{2}$ is then a quotient $\Gamma \backslash X$, where $X \cong \mathbb{R}^{4}$ is taken from the list above and $\Gamma$ is a discrete subgroup which acts freely on $X$. Although $\Gamma$ is not necessarily a subgroup of $X$, it always contains a subgroup $\Gamma_{0}$, which is a lattice of $X$, such that $\Gamma_{0} \backslash \Gamma$ is finite. As such, $\Gamma \backslash X$ is covered by the solvmanifold $\Gamma_{0} \backslash X$. When $\Gamma=\Gamma_{0}$ the quotient $\Gamma \backslash X$ is a solvmanifold (resp. nilmanifold if $X$ is nilpotent), otherwise $\Gamma \backslash X$ is called an infra-solvmanifold (resp. infra-nilmanifold). In the list above, in the cases $\left(1_{\mathrm{a}}\right)$ and $(2)$, the group $\Gamma$ is always a lattice, while in the case $\left(3_{\mathrm{a}}\right) \Gamma$ is a lattice if and only if its last generator has $\epsilon=1$. In the cases $\left(1_{\mathrm{b}}\right)$ and $\left(1_{\mathrm{c}}\right)$ the quotient $\Gamma \backslash X$ is an infra-nilmanifold such that $\Gamma_{0} \backslash \Gamma=\mathbb{Z}_{2}$, and in $\left(3_{\mathrm{b}}\right) \Gamma$ is not a lattice of $X$. 
Example 2.1. In case ( $\left.3_{\mathrm{a}}\right)$, take $\alpha_{4}=\beta_{4}=0$ and $\epsilon=1$. We also take $\alpha_{3}=\beta_{3}=0$ so that $M=M^{3} \times S^{1}$ is the product of a solv-3-manifold with a circle. The lattice in $\mathbb{R}^{2}$ defining $\mathbb{T}^{2}$ will be generated by

$$
\left(\begin{array}{c}
\alpha_{1} \\
\beta_{1}
\end{array}\right)=\left(\begin{array}{l}
1 \\
1
\end{array}\right), \quad\left(\begin{array}{c}
\alpha_{2} \\
\beta_{2}
\end{array}\right)=\left(\begin{array}{c}
\sigma \\
\sigma^{-1}
\end{array}\right),
$$

where $\sigma$ and $\sigma^{-1}$ are the two roots of the quadratic equation $\sigma^{2}-n \sigma+1=0$, with $n=3,4,5$. Setting $\delta=\log \sigma$ ensures that

$$
\left(\begin{array}{cc}
\mathrm{e}^{\delta} & 0 \\
0 & \mathrm{e}^{-\delta}
\end{array}\right)=\left(\begin{array}{cc}
1 & \sigma \\
1 & \sigma^{-1}
\end{array}\right)\left(\begin{array}{cc}
0 & -1 \\
1 & n
\end{array}\right)\left(\begin{array}{cc}
1 & \sigma \\
1 & \sigma^{-1}
\end{array}\right)^{-1},
$$

so $\Gamma$ is closed under multiplication. The example on [4, page 25] is the instance $n=3$.

The inequivalent fibrations $\pi: M \rightarrow \mathbb{T}^{2}$ are induced from the following coordinate mappings:

$$
(x, y, z, t) \mapsto\left\{\begin{array}{cl}
(x, y) \text { or }(y, t) & \text { for }\left(1_{\mathrm{a}}\right), \\
(y, t) & \text { for }\left(1_{\mathrm{b}}\right),\left(1_{\mathrm{c}}\right), \\
(z, t) & \text { for }(2),\left(3_{\mathrm{a}}\right) \text { and }\left(3_{\mathrm{b}}\right) .
\end{array}\right.
$$

In view of [9, Theorem 1], the total space $M$ always admits a symplectic structure, and we shall check this case by case early in the next section.

\section{Almost-Kähler structures}

Since our main focus of attention is real symplectic geometry, we first discuss the existence of invariant closed non-degenerate 2-forms compatible with each of the fibrations listed in (2.3) . Any such 2 -form can always be expressed as $f^{13}+f^{42}$ relative to some basis $\left(f^{i}\right)$ related by a constant linear transformation to a basis $\left(e^{i}\right)$ defined by (2.3). It follows that we can choose a Riemannian metric $g$ (for example $\sum f^{i} \otimes f^{i}$ ) and an orthogonal almost-complex structure $J$ (for example,

$$
\left.J=f^{1} \otimes f_{3}-f^{3} \otimes f_{1}-f^{2} \otimes f_{4}+f^{4} \otimes f_{2}\right),
$$

such that

$$
\Omega(X, Y)=g(J X, Y) .
$$

The resulting triple $(g, J, \Omega)$ constitutes an invariant almost-Kähler structure on $M$.

Returning to (2.3), we first remark that $M$ has a complex structure if and only if $b_{1}(M)=3$, which is precisely case $\left(1_{\mathrm{a}}\right)$ of the previous section 9 . We shall denote the two inequivalent projections from the first row of (2.5) by

$$
\begin{array}{ll}
\pi_{x y}: & M \rightarrow \mathbb{T}_{x y}^{2}, \\
\pi_{y t} & : \quad M \rightarrow \mathbb{T}_{y t}^{2} .
\end{array}
$$

The usual projection $\pi_{x y}$ defining the Kodaira-Thurston manifold $M$ is holomorphic with respect to an invariant complex structure $J_{1}$. There is also a holomorphic symplectic 2 -form $\Theta=\Omega_{2}+i \Omega_{3}$ for which $\pi_{x y}$ is bi-Lagrangian, meaning that both $\Omega_{2}$ and $\Omega_{3}$ restrict to zero on the fibres. This is equivalent to the single complex equation $\Theta \wedge e^{12}=0$. With the labelling below, the canonical choice is $\Omega_{2}=e^{13}+e^{42}$ and $\Omega_{3}=e^{14}+e^{23}$ (see (4.1) and (4.2) ), though one aspect of Section 4 is to explain that there are families of equivalent such structures. 
With the above convention, $\Omega_{2} \wedge e^{13} \neq 0$, so $\Omega_{2}$ is non-degenerate on the fibres of $\pi_{y t}$. Indeed, $\Omega_{2}$ is compatible with the almost-complex structure characterized by

$$
J e^{1}=e^{3}, \quad J e^{4}=e^{2},
$$

for which $\pi_{y t}$ is holomorphic. By this we mean that $J$ induces a complex structure $\mathbb{J}$ on $\mathbb{T}=\mathbb{T}_{y t}^{2}$ and the differential

$$
\pi_{*}:\left(T_{m} M, J\right) \rightarrow(\mathbb{T}, \mathbb{J})
$$

is complex linear ( $\mathbb{J}$ is necessarily integrable for dimensional reasons). This situation, in which the projection respects the almost-Kähler structure, contrasts with that for $\pi_{x y}$. On the other hand, the fibres of $\pi_{y t}$ remain Lagrangian for $\Omega_{3}$. We shall study $\left(M, \Omega_{3}\right)$ as a limiting case of the $N i l^{4}$ model, in which $e^{13}$ is again the volume form on the base and $\Omega_{3}$ is a symplectic form for which the fibres are necessarily Lagrangian.

In the cases different from $\left(1_{\mathrm{a}}\right)$, the fibration of $M$ as a torus bundle is unique [9, Theorem 1]. In $\left(3_{\mathrm{a}}\right)$ and $\left(3_{\mathrm{b}}\right)$, the symplectic form $e^{12}+e^{34}$ is non-zero (and so non-degenerate) on the fibres of $\pi_{z t}$. Note that $e^{34}$ is closed by (2.3) (3), in which the distinction between $e^{41}$ and $e^{14}$ is important. The following general result is relevant to this situation.

Proposition 3.1. Let $\Omega$ be an invariant symplectic form on $M=\Gamma \backslash X$, and suppose that $\Omega$ is non-zero on the fibres of a $T^{2}$-fibration $\pi: M \rightarrow \mathbb{T}^{2}$. Then there exists an invariant almost-Kähler structure $(g, J, \Omega)$ on $M$ for which $\pi$ is $J$-holomorphic. In this situation, if $\sigma=\mathrm{e}^{F} \Omega^{2}$ is a volume form satisfying (1.1) with $F \in C^{\infty}\left(\mathbb{T}^{2}\right)$, the associated Calabi-Yau problem has a solution.

Proof. Let $f^{12}$ be a volume form on the base $\mathbb{T}^{2}$, and fix a point $m \in M$. By assumption, $f^{12} \wedge \Omega \neq 0$, and we can choose $\mu \in \mathbb{R}$ such that

$$
0=\left(\Omega-\mu f^{12}\right)^{2}=\Omega^{2}-2 \mu f^{12} \wedge \Omega
$$

at $m$. This means that $\Omega-\mu f^{12}$ is a simple 2 -form, and so there exists a basis $\left(f^{i}\right)$ of 1 -forms such that

$$
\Omega=\mu f^{12}+f^{34} .
$$

The required almost-Kähler structure is obtained by defining $J$ to be the almostcomplex structure $J$ whose value at $m$ is given by $J f_{1}=\mu f_{2}$ and $J f_{3}=f_{4}$ in the dual basis. As above, $J$ induces a complex structure $\mathbb{J}$ on $\mathbb{T}^{2}$, and (3.2) is complex linear.

As $m$ varies along the fibre, the parameter $\mu$ remains constant by invariance. To solve the Calabi-Yau problem, we merely set

$$
\tilde{\Omega}=\mathrm{e}^{F} \mu f^{12}+f^{34},
$$

which yields (1.2) immediately. Observe that $\tilde{\Omega}-\Omega$ is the pullback of the $(1,1)$ form $\left(\mathrm{e}^{F}-1\right) \mu f^{12}$ on $\mathbb{T}=\mathbb{T}^{2}$. It remains to show that it evaluates to zero on the fundamental cycle $[\mathbb{T}] \in H_{2}(\mathbb{T}, \mathbb{Z})$, but

$$
\int_{\mathbb{T}}\left(\mathrm{e}^{F}-1\right) \mu f^{12}=\int_{M}\left(\mathrm{e}^{F}-1\right) \mu f^{1234}=\frac{1}{2} \int_{M}\left(\sigma-\Omega^{2}\right)=0,
$$

by hypothesis. 
For completeness, we reproduce the "negative chords" argument from [5] that guarantees that the elementary solution above is unique. We shall tag this uniqueness statement to all our own existence results without further comment (except for Remark 4.6).

Proposition 3.2. Any solution of the Calabi-Yau problem (1.1), (1.2), (1.3) on a compact 4-manifold is unique.

Proof. Given two solutions $\tilde{\Omega}, \tilde{\Omega}^{\prime}$ of the problem, set $\tilde{\Omega}-\tilde{\Omega}^{\prime}=d \alpha$ and $\tilde{\Omega}+\tilde{\Omega}^{\prime}=\widehat{\Omega}$. With respect to $J$, both $d \alpha$ and $\widehat{\Omega}$ are of type $(1,1)$ and the latter defines a (positivedefinite) Riemannian metric $\widehat{g}$ by the usual formula $\widehat{\Omega}(X, Y)=\widehat{g}(J X, Y)$. Relative to $\widehat{g}$, we have the decomposition

$$
\bigwedge^{2} T_{m}^{*} M=\Lambda^{+} \oplus \Lambda^{-}
$$

into self-dual and anti-self-dual forms at each point, and the (1,1)-forms are generated by $\Lambda^{-}$together with $\widehat{\Omega}$. But since $\widehat{\Omega} \wedge d \alpha=0$, we can conclude that $d \alpha \in \Lambda^{+}$, and $d \alpha \wedge d \alpha$ is $\|d \alpha\|^{2}$ times $\operatorname{vol}_{\widehat{g}}$. A standard Stokes' argument then tells us that $d \alpha=0$.

3.1. The condition $\mathcal{R}>0$. Tosatti, Weinkove and Yau defined a certain tensor $\mathcal{R}$ using the canonical connection and Nijenhuis tensor of $J[23$. They proved that if $\mathcal{R}$ is positive, then the Calabi-Yau equation has a solution.

On any almost-Kähler manifold $(M, J, \Omega)$ there exists a unique connection $\nabla$ such that $\nabla J=0, \nabla \Omega=0$ and whose torsion has no $(1,1)$-component. This is the so-called canonical connection or Chern connection (though the latter is sometimes reserved for the Hermitian case). We denote its curvature tensor by $R$, and set

$$
\mathcal{R}_{i \bar{j} k \bar{l}}(g, J)=R_{i k \bar{l}}^{j}+4 N_{\bar{l} \bar{j}}^{r} \overline{N_{\bar{r}}^{i} \bar{k}},
$$

where $N$ is the Nijenhuis tensor of $J$. According to [23], the condition

$$
\mathcal{R}(g, J) \geqslant 0,
$$

implies that the Calabi-Yau equation can be solved for every normalized volume form.

It was pointed out in 25] that (3.5) fails to hold for the Kodaira-Thurston manifold. In fact, it is never satisfied under the hypothesis of Theorem 1.1. To see this, we first prove

Proposition 3.3. Let $(M, J, \Omega)$ be a 4-dimensional almost-Kähler manifold, and let $s=g^{r \bar{k}} g^{s \bar{l}} R_{r \bar{k} s \bar{l}}$ be the scalar curvature of $\nabla$. Assume $s \leqslant 0$. Then condition (3.5) is satisfied if and only if $(J, \Omega)$ is a Kähler structure.

Proof. Let $Z_{1}, Z_{2}$ be a local unitary frame on $M$. Then direct computation gives

$$
\begin{aligned}
& \mathcal{R}_{1 \overline{1} 1 \overline{1}}=R_{11 \overline{1}}^{1}, \\
& \mathcal{R}_{2 \overline{2} 2 \overline{2}}=R_{22 \overline{2}}^{2}, \\
& \mathcal{R}_{1 \overline{1} 2 \overline{2}}=R_{12 \overline{2}}^{1}+4 N_{\overline{2} \overline{1}}^{1} N_{12}^{\overline{1}}=R_{21 \overline{1}}^{2}-4\left|N_{\overline{1} \overline{2}}^{1}\right|^{2}, \\
& \mathcal{R}_{2 \overline{2} 1 \overline{1}}=R_{21 \overline{1}}^{2}+4 N_{\overline{1} \overline{2}}^{2} N_{21}^{\overline{2}}=R_{21 \overline{1}}^{2}-4\left|N_{\overline{1} \overline{2}}^{2}\right|^{2} .
\end{aligned}
$$

These equations imply

$$
\begin{aligned}
& \mathcal{R}_{2 \overline{2} 2 \overline{2}}+\mathcal{R}_{1 \overline{1} 2 \overline{2}}=\operatorname{Ric}(J)_{2 \overline{2}}-4\left|N_{\overline{1} \overline{2}}^{1}\right|^{2}, \\
& \mathcal{R}_{1 \overline{1} 1 \overline{1}}+\mathcal{R}_{2 \overline{2} 1 \overline{1}}=\operatorname{Ric}(J)_{1 \overline{1}}-4\left|N_{\overline{1} \overline{2}}^{2}\right|^{2},
\end{aligned}
$$


and

$$
\mathcal{R}_{2 \overline{2} 2 \overline{2}}+\mathcal{R}_{1 \overline{1} 2 \overline{2}}+\mathcal{R}_{1 \overline{1} 1 \overline{1}}+\mathcal{R}_{2 \overline{2} 1 \overline{1}}=s-4\left|N_{\overline{1} \overline{2}}^{1}\right|^{2}-4\left|N_{\overline{1} \overline{2}}^{2}\right|^{2} .
$$

Hence if $s$ is non-positive and condition (3.5) is satisfied, then $N$ vanishes.

Corollary 3.4. On a 4-dimensional infra-solvmanifold $M=\Gamma \backslash G$ with an invariant almost-Kähler structure, condition (3.5) is satisfied only in the Kähler case.

Proof. We first prove the statement in the solvable case when $\Gamma$ is a lattice of $G$. If $J$ is an invariant almost-complex structure, then its first Chern class vanishes. This implies that the Ricci form $\rho$ associated to any $J$-compatible metric $g$ is exact. If $g$ is invariant, then also $\rho$ is left-invariant and the scalar curvature $s=\operatorname{tr}_{g} \rho$ is constant. Therefore if $g$ is an invariant $J$ compatible almost-Kähler metric with associated symplectic form $\omega$, we have

$$
0=\int_{M} \rho \wedge \omega=c s \int_{M} \omega^{2},
$$

where $c$ is a non-zero constant. Hence $s$ vanishes and the claim follows.

On the other hand, if $M$ is an infra-solvmanifold with an invariant almost-Kähler structure, then it is covered by an almost-Kähler solvmanifold. Since the tensor $\mathcal{R}$ depends only from the almost-Kähler structure, then condition (3.5) is invariant for covering, which preserves the almost-Kähler structure and the claim follows.

\section{Manifolds mOdelled ON $N i l^{3} \times \mathbb{R}$}

In this section, we consider in more detail the Kodaira-Thurston manifold $M=$ $\Gamma_{0} \backslash X$, a discrete quotient of $X=N i l^{3} \times \mathbb{R}$. The standard choice of $\Gamma_{0}$ (case $\left(1_{\mathrm{a}}\right)$ of Section 2 with $\lambda=1$ ) allows us to consider the two principal $T^{2}$-fibrations (3.1) with 2-torus base.

The manifold $M$ has a global basis consisting of real 1-forms

$$
e^{1}=d y, \quad e^{2}=d x, \quad e^{3}=d t, \quad e^{4}=d z-x d y .
$$

This basis conforms to the first structure equation in (2.3), although the 1-dimensional factor in $\mathrm{Nil}^{3} \times \mathbb{R}$ is in third place. Any tensor that can be expressed in terms of it with constant coefficients is called invariant to reflect the fact that it is induced from a left-invariant tensor on the Lie group $X$. In particular, $M$ inherits a complex structure and holomorphic symplectic form

$$
\left(e^{1}+i e^{2}\right) \wedge\left(e^{3}+i e^{4}\right)=d \zeta_{1} \wedge d \zeta_{2},
$$

where

$$
\zeta_{1}=y+i x, \quad \zeta_{2}=t-\frac{1}{2} x^{2}+i z
$$

are local complex coordinates on the base and fibres. The latter become biLagrangian, and $\pi_{x y}$ is holomorphic. This observation is readily generalized.

We first describe the space of invariant symplectic forms on $M$. An element of this space lies in

$$
\operatorname{ker} d=\left\langle e^{12}, e^{13}, e^{14}, e^{23}, e^{24}\right\rangle
$$

and so equals

$$
\Omega_{\lambda, A}=\lambda e^{12}+e^{1} \wedge\left(a e^{3}+b e^{4}\right)-e^{2} \wedge\left(c e^{3}+d e^{4}\right),
$$


for some $\lambda \in \mathbb{R}$ and some real $2 \times 2$ matrix $A=\left(\begin{array}{ll}a & b \\ c & d\end{array}\right)$ with $\operatorname{det} A \neq 0$. With this convention, (4.2) equals $\Omega_{0, I}+i \Omega_{0, J}$, where $I$ is the identity matrix and $J=$ $\left(\begin{array}{cc}0 & 1 \\ -1 & 0\end{array}\right)$.

Lemma 4.1. The fibration $\pi_{x y}$ is Lagrangian with respect to any invariant symplectic form on $M$.

Proof. The simple 2-form $e^{12}$ represents a non-zero volume form on the base of $\pi_{x y}$. At the level of differential forms, the restriction of $\Omega_{\lambda, A}$ to any fibre is therefore determined by the wedge product $\Omega_{\lambda, A} \wedge e^{12}=0$. The result follows.

More generally, consider two such forms $\Omega_{2}, \Omega_{3}$ with $\Omega_{i}=\Omega_{\lambda_{i}, A_{i}}$. It is not difficult to arrange for $\Theta=\Omega_{2}+i \Omega_{3}$ to satisfy $\Theta \wedge \Theta=0$; this is equivalent to asserting

$$
\operatorname{det}(B)=1, \quad \operatorname{tr}(B)=0, \quad B=A_{2}^{-1} A_{3} .
$$

When conditions (4.3) hold, we can write $\Theta=\alpha_{1} \wedge \alpha_{2}$ over $\mathbb{C}$, whence $\Lambda^{1,0}=$ $\left\langle\alpha_{1}, \alpha_{2}\right\rangle$ is the space of $(1,0)$-forms relative to some invariant complex structure $J_{1}$ on $M$. Observe that

$$
0=d \Theta=d \alpha_{1} \wedge \alpha_{2}-\alpha_{1} \wedge d \alpha_{2},
$$

which proves that $\left(d \alpha_{i}\right)^{0,2}=0$ for $i=1,2$. The integrability of $J_{1}$ then follows from the Newlander-Nirenberg theorem.

Since $\Theta \wedge e^{12}=0$, the real 2 -form $e^{12}$ has type $(1,0)$ relative to $J_{1}$. It follows that we can choose $p, q \in \mathbb{C}$ so that $\alpha_{1}=p e^{1}+q e^{2}$, defining a complex structure on the base $\mathbb{T}_{x y}$ relative to which $\pi_{x y}$ is holomorphic. The fibres of $\pi_{x y}$ themselves become holomorphic curves in $\left(M, J_{1}\right)$. We complete these observations with

Proposition 4.2. Given an invariant almost-Kähler structure $\left(g, J_{2}, \Omega_{2}\right)$ on $M$, we can choose a holomorphic symplectic structure $\Theta=\Omega_{2}+i \Omega_{3}$ and associated complex structure $J_{1}$ for which $\pi_{x y}$ is holomorphic. Conversely, any invariant complex structure on $M$ arises in this way.

Proof. By assumption, $g$ is a scalar product for which

$$
g\left(J_{2} X, J_{2} Y\right)=g(X, Y), \quad g\left(J_{2} X, Y\right)=\Omega_{2}(X, Y) .
$$

Orient $M$ by means of $J_{2}$, so that the 3-dimensional space $\Lambda^{+}$of self-dual 2-forms relative to $g$ contains the closed 2 -form $\Omega_{2}$. Since ker $d$ has dimension 5 on the space of 2 -forms, we can find a non-zero closed 2-form $\Omega_{3}$ in the orthogonal complement of $\Omega_{2}$ in $\Lambda^{+}$. The orthogonality means that $\Omega_{2} \wedge \Omega_{3}=0$, and we can normalize $\Omega_{3}$ so that $\Omega_{2} \wedge \Omega_{2}=\Omega_{3} \wedge \Omega_{3}$. It follows that $\Theta=\Omega_{2}+i \Omega_{3}$ satisfies $\Theta \wedge \Theta=0$ as in (4.3), and $\pi_{x y}$ is holomorphic.

Conversely, suppose that $J$ is an arbitrary invariant complex structure. Since $J$ is integrable, its space $\Lambda^{1,0}$ of $(1,0)$-forms is generated by invariant 1-forms $\alpha_{1}, \alpha_{2}$ for which $d \alpha_{1}=0$ and $d \alpha_{2}$ is divisible by $\alpha_{1}$, by [20. Theorem 1.3]. It follows that the invariant 2-form $\alpha_{1} \wedge \alpha_{2} \in \Lambda^{2,0}$ is closed and, after fixing a compatible metric, we have recovered the initial hypothesis.

Remark 4.3. The discussion above shows that $M$ possesses a non-trivial moduli space of holomorphic symplectic structures $\Theta$. Specifying the invariant complex structure $J_{1}$ is equivalent to specifying the projective class $\langle\Theta\rangle$, and $J_{1}$ in turn 
determines a complex structure on both the base $\mathbb{T}_{x y}^{2}$ and a fibre $T^{2}$ that become elliptic curves. Taken together, these two complex structures can be specified by a pair of invariants $\left(j_{1}, j_{2}\right) \in \mathbb{C}^{2}$. A third parameter is required to 'scale' base and fibre, but can be eliminated by quotienting by the action of right translation by $N i l^{3}$ commuting with $\Gamma$; cf. [13, 18].

Given an invariant almost-Kähler structure, extended as in the proposition, it is possible to set $\Theta=\alpha_{1} \wedge \alpha_{2}$, where

$$
\alpha_{1}=f^{1}+i f^{2} \in\left\langle e^{1}, e^{2}\right\rangle_{\mathbb{C}}, \quad \alpha_{2}=f^{3}+i f^{4},
$$

the $f^{i}$ have equal norms relative to $g$, and $d f^{i}=0$ for $i=1,2,3$. (To achieve the latter, multiply $\alpha_{2}$ by a unit complex number so that $f^{3}$ is a linear combination of $e^{1}, e^{2}, e^{3}$.) We can now write $d f^{4}=k f^{12}$ with $k \neq 0$. Apart from this last factor, we have recovered the structure equation (2.3) (1). In particular, any two invariant holomorphic symplectic structures are equivalent by a Lie algebra automorphism.

With the assumptions of the last paragraph, the space $\Lambda^{+}$of self-dual 2 -forms (see (3.4) ) is generated by

$$
\Omega_{1}=f^{12}+f^{34}, \quad \Omega_{2}=f^{13}+f^{42}, \quad \Omega_{3}=f^{14}+f^{23} .
$$

The space $\Lambda^{+}$determines the conformal (or rather, in an invariant context, the homothety) class of $g=\sum f^{i} \otimes f^{i}$. Its annihilator $\Lambda^{-}=\left\langle\Omega_{1}, \Omega_{2}, \Omega_{3}\right\rangle^{\circ}$ (relative to wedge product) is the space of anti-self-dual 2 -forms.

We shall in fact fix the homothety class of $g$ by requiring that

$$
\Theta=\left(f^{1}+i f^{2}\right) \wedge\left(f^{3}+i f^{4}\right)=\Omega_{2}+i \Omega_{3}
$$

have the same norm as (4.2). The forms (4.4) are therefore characterized by the conditions

$$
\left\{\begin{array}{l}
\Omega_{i} \wedge \Omega_{j}=0, \quad i \neq j \\
\Omega_{i} \wedge \Omega_{i}=2 e^{1234}, \quad i=1,2,3 \\
d \Omega_{i}=0, \quad i=2,3
\end{array}\right.
$$

Setting $\Omega_{i}(v, w)=g\left(J_{i} v, w\right)$ extends the definition on $J_{1}$ into a triple $J_{1}, J_{2}, J_{3}$ of almost-complex structures.

The main result of this section is

Theorem 4.4. Let $F \in C^{\infty}(\mathbb{T})$ be a smooth function on the base $\mathbb{T}=\mathbb{T}_{x y}^{2}$ such that

$$
\int_{\mathbb{T}}\left(\mathrm{e}^{F}-1\right)=0
$$

Fix a triple of 2-forms (4.4) satisfying (4.6), and set $\Theta=\Omega_{2}+i \Omega_{3}$. Then there exists a closed complex-valued 2-form $\widetilde{\Theta}$ on $M$ such that

- $\widetilde{\Theta} \wedge \widetilde{\Theta}=0$ and $\widetilde{\Theta} \wedge \widetilde{\widetilde{\Theta}}=4 \mathrm{e}^{F} e^{1234}$;

- $\widetilde{\Theta}=h \Theta+\Phi_{-}$, where $h \in C^{\infty}(\mathbb{T})$ and $\Phi_{-}: \mathbb{T} \rightarrow\left(\Lambda_{-}^{2}\right)_{\mathbb{C}}$;

- $[\widetilde{\Theta}]=[\Theta]$ in $H^{2}(M, \mathbb{C})$.

Proof. For simplicity, we first suppose that $f^{1}=e^{1}=d x$ and $f^{2}=e^{2}=d y$, which is the case when $\Theta$ has the standard form (4.2). Consider the 2-dimensional Monge-Ampère equation

$$
P_{x x} P_{y y}-P_{x y}^{2}=\mathrm{e}^{F}
$$


where

$$
P(x, y)=\frac{1}{2}\left(x^{2}+y^{2}\right)+p(x, y),
$$

with $p \in C^{\infty}(\mathbb{T})$. This equation can be rewritten

$$
d P_{x} \wedge d P_{y}=\mathrm{e}^{F} f^{12}
$$

On the other hand, substituting (4.9), $\int_{\mathbb{T}} d P_{x} \wedge d P_{y}=\int_{\mathbb{T}} f^{12}$, since $p$ and its derivatives integrate to zero. Thus, (4.7) is a necessary condition for a solution to (4.10).

We define

$$
\widetilde{\Theta}=\left(d P_{x}+i d P_{y}\right) \wedge\left(f^{3}+i f^{4}\right),
$$

in analogy to (4.5). The first conditions

$$
\begin{gathered}
\widetilde{\Theta} \wedge \widetilde{\Theta}=0, \\
\widetilde{\Theta} \wedge \widetilde{\widetilde{\Theta}}=4 d P_{x} \wedge d P_{y} \wedge f^{34}=4 \mathrm{e}^{F} e^{1234},
\end{gathered}
$$

are then immediate consequences of (4.11) and (4.10).

Recalling that $\Omega_{1}=f^{12}+f^{34}$, it is equally obvious that $\widetilde{\Theta} \wedge \Omega_{1}=0$. We also have $\widetilde{\Theta} \wedge \Theta=0$, and, with a bit more work,

$$
\widetilde{\Theta} \wedge \bar{\Theta}=2\left(P_{x x}+P_{y y}\right) \text {. }
$$

The second bullet point follows by setting $h=\frac{1}{2}\left(P_{x x}+P_{y y}\right)$.

Finally, we may write

$$
\begin{aligned}
\widetilde{\Theta}-\Theta & =\left(d p_{x}+i d p_{y}\right) \wedge\left(f^{3}+i f^{4}\right) \\
& =d\left[\left(p_{x}+i p_{y}\right) \wedge\left(f^{3}+i f^{4}\right)\right]-\left(p_{x}+i p_{y}\right) \wedge i k f^{12} \\
& =d \alpha
\end{aligned}
$$

where

$$
\alpha=-k p\left(f^{1}+i f^{2}\right)+\left(p_{x}+i p_{y}\right)\left(f^{3}+i f^{4}\right)
$$

is a well-defined 1 -form on $\mathbb{T}$. Note that we have used the assumption $d f^{4}=k f^{12}$ reflecting the arbitrary invariant metric at the start.

To know that we can solve (4.8), it suffices to take $n=2, S_{i j}=\delta_{i j}$ and $G=F$ in the following theorem, taken from [16].

Let $\mathbb{T}^{n}=\mathbb{R}^{n} / \mathbb{Z}^{n}$ be a torus with flat coordinates $x_{i}$. Suppose that $F \in C^{\infty}\left(\mathbb{T}^{n}\right)$ is a positive function and that $\left(S_{i j}\right)$ is a symmetric positive-definite $n \times n$ matrix satisfying

$$
\int_{\mathbb{T}^{n}}\left(F-\operatorname{det}\left(S_{i j}\right)\right)=0 .
$$

Consider the equation

$$
\operatorname{det}\left(S_{i j}+\frac{\partial^{2} p}{\partial x_{i} \partial x_{j}}\right)=\mathrm{e}^{G} \quad \text { on } \quad \mathbb{T}^{n}
$$

together with the positive-definite condition

$$
\left(S_{i j}+\frac{\partial^{2} p}{\partial x_{i} \partial x_{j}}\right)>0 \quad \text { on } \mathbb{T}^{n} .
$$

Then there exists $p \in C^{\infty}\left(\mathbb{T}^{n}\right)$ satisfying (4.14) and (4.15), and $p$ is unique up to addition of a constant. Moreover, (4.13) is necessary for the solvability of (4.14). 
In general, there will be a constant matrix $A=\left(A_{i j}\right)$ such that $e^{i}=\sum_{i=1}^{2} A_{i j} f^{j}$. We therefore make a linear substitution of coordinates

$$
x=A_{11} u+A_{12} v, \quad y=A_{21} u+A_{22} v,
$$

so that

$$
f^{1}=d u, \quad f^{2}=d v .
$$

This allows us to proceed as before; the differential equations to be solved are the same except that all derivatives with respect to $x, y$ are replaced by those with respect to $u, v$. Since

$$
H=\left(\begin{array}{cc}
P_{u u} & P_{u v} \\
P_{u v} & P_{v v}
\end{array}\right)=I+A^{\top}\left(P_{x_{i} x_{j}}\right) A,
$$

the Monge-Ampère equation $\operatorname{det} H=\mathrm{e}^{F}$ in the new coordinates takes the form (4.14) with

$$
\left(S_{i j}\right)=\left(A A^{\top}\right)^{-1}, \quad \mathrm{e}^{G}=\mathrm{e}^{F} /(\operatorname{det} A)^{2} .
$$

The theorem then implies the existence $p \in \mathbb{C}^{\infty}\left(\mathbb{T}^{2}\right)$ solving (4.7).

Taking the real part of the second bullet point yields

$$
\widetilde{\Omega}_{2}=h \Omega_{2}+\Phi_{2},
$$

where $\Phi_{2}$ is a $(1,1)$-form relative to all of $J_{1}, J_{2}, J_{3}$. In particular,

$$
d(\operatorname{Re} \alpha)=\widetilde{\Omega}_{2}-\Omega_{2}=(h-1) \Omega_{2}+\Phi_{2}
$$

is a $(1,1)$-form relative to $J_{2}$. Moreover, the new structure has the assigned volume form

$$
\widetilde{\Omega}_{2} \wedge \widetilde{\Omega}_{2}=\mathrm{e}^{F} \Omega_{2} \wedge \Omega_{2}
$$

In conclusion,

Corollary 4.5. Given an invariant almost-Kähler structure $\left(g, J_{2}, \Omega_{2}\right)$ on $M$, the associated Calabi-Yau problem admits a unique solution.

Remark 4.6. The uniqueness statement for solutions of Monge-Ampère on the 2torus base will translate into a uniqueness result for the 4-dimensional problem. The only gap in our presentation is the proof that the problem on $M$ necessarily gives rise to a solution of (4.8). However, uniqueness is already covered by Proposition 3.2. whose proof translates directly into the following neat argument for (4.8).

Taking

$$
2 h=P_{u u}+P_{v v}=4+\Delta p,
$$

to be positive, the triple $\left(P_{u u}, P_{v v}, P_{u v}\right)$ defines (at each point of $\mathbb{T}$ ) a vector inside the forward light cone in $\mathbb{R}^{1,2}$ with a Lorentzian norm equal to $\mathrm{e}^{F}$. If $P, \widetilde{P}$ are two solutions, set

$$
P^{\prime}=P-\widetilde{P}, \quad P^{\prime \prime}=P+\widetilde{P} .
$$

Then $\left(P_{u u}^{\prime}, P_{v v}^{\prime}, P_{u v}^{\prime}\right)$ (having zero Lorentzian product with $\left(P_{u u}^{\prime \prime}, P_{v v}^{\prime \prime}, P_{u v}^{\prime \prime}\right)$ ) is everywhere spacelike or zero, so $d P_{u}^{\prime} \wedge d P_{v}^{\prime}$ is a non-negative multiple of $e^{12}$. But $P^{\prime}$ is biperiodic, so the integral of this 2 -form over $\mathbb{T}^{2}$ vanishes and $P^{\prime}$ must be constant. 
4.1. The potential. To further understand the proof of Theorem 4.4, note that $\widetilde{\Theta}$, by its very definition (4.11), is holomorphic relative to the complex structure with local coordinate $P_{u}+i P_{v}$ on the base and equal to $J_{1}$ on the fibres. In this sense, $P$ is a potential function for the modified complex structure, and the assigned volume form on $\mathbb{T}^{2}$ then determines the Hessian metric

$$
d s^{2}=P_{u u} d u^{2}+2 P_{u v} d u d v+P_{v v} d v^{2},
$$

represented by the matrix $H$ of (4.16). This fundamental form is then duplicated on the fibres of $M$. Such metrics are well known in work on special Lagrangian and toric geometry in a Kähler setting [12, 2].

The potential $P$ does not feature in [25], but is an integral of functions considered there. Nonetheless, the Tosatti-Weinkove estimate

$$
\tilde{\Delta} h \geqslant \inf _{\mathbb{T}^{2}} \Delta F
$$

makes sense in the above context, where $\tilde{\Delta}$ is the Laplacian relative to (4.17), and their proof of it translates into the following. Take derivatives of (4.8), and rearrange them to give an equation of the form

$$
\Delta F=\Delta_{\text {even }}-\Delta_{\text {odd }},
$$

where $\Delta_{\text {even }}$ (resp. $\Delta_{\text {odd }}$ ) involves products of partial derivatives of even (resp. odd) total order. Moreover,

$$
\begin{aligned}
& \Delta_{\text {even }}=\operatorname{tr}\left(H^{-1} \operatorname{Hess} h\right) \tilde{\Delta} h, \\
& \Delta_{\text {odd }}=\wp\left(H^{-1} H_{u}\right)+\wp\left(H^{-1} H_{v}\right),
\end{aligned}
$$

$\wp(B)=(\operatorname{tr} B)^{2}-2 \operatorname{det} B \geqslant 0$ for $B$ a symmetric matrix.

4.2. Other manifolds belonging to the family $\left(1_{\mathrm{a}}\right)$. All the computations we have performed in this section on the Kodaira-Thurston manifold can be used for every manifold $M=\Gamma \backslash\left(N i l^{3} \times \mathbb{R}\right)$ belonging to class $\left(1_{\mathrm{a}}\right)$. Every $\Gamma$ is a lattice of $N i l^{3} \times \mathbb{R}$, and the quotient $M$ is a genuine nilmanifold.

\section{MANifolds MOdelled ON $\mathrm{Nil}^{4}$}

Let $M$ be the total space of a torus bundle belonging to case (2) of Section 2 , so that $M$ is a nilmanifold associated to the 3 -step nilpotent Lie group (2.2). The third row of (2.5) yields a projection

$$
\pi_{z t}: M \rightarrow \mathbb{T}_{z t}^{2}
$$

with $T^{2}$ fibres. We adopt the following basis of left-invariant vector fields,

$$
e_{1}=-\partial_{t}, \quad e_{2}=\partial_{y}+t \partial_{x}, \quad e_{3}=\partial_{z}+t \partial_{y}+\frac{1}{2} t^{2} \partial_{x}, \quad e_{4}=\partial_{x},
$$

and dual basis of 1 -forms,

$$
e^{1}=-d t, \quad e^{2}=d y-t d z, \quad e^{3}=d z, \quad e^{4}=d x-t d y+\frac{1}{2} t^{2} d z .
$$

With this convention, $\left(e^{i}\right)$ satisfies (2.4), which coincides with setting $\lambda=1$ in (5.4) in the next lemma.

The analogue of Lemma 4.1 is valid for $\pi_{z t}$. This is because any symplectic form $\Omega$ belongs to

$$
\text { ker } d=\left\langle e^{12}, e^{13}, e^{14}, e^{23}\right\rangle .
$$


This time it is $e^{13}=d z \wedge d t$ that represents a volume form on the base, and

$$
\Omega \wedge e^{13}=0 .
$$

Of course, it remains true that $\Omega \wedge e^{12}=0$.

The canonical choice for a positively oriented symplectic form is $e^{14}+e^{23}$, and the next result shows that the general case is not so very different. We state it in a form that will also serve in Section 7

Lemma 5.1. Let $(g, J, \Omega)$ be an invariant almost-Kähler structure on a 4-dimensional nilmanifold that satisfies

$$
d e^{1}=0, \quad d e^{2}=\lambda e^{13}, \quad d e^{3}=0, \quad d e^{4}=e^{12},
$$

with $\lambda \in \mathbb{R}$. Assume (5.3) (which is an extra hypothesis only if $\lambda=0$ ). There exists an orthonormal basis $\left(f^{i}\right)$ for which 1

$$
\Omega=f^{14}+f^{23}
$$

and

$$
f^{1} \in\left\langle e^{1}\right\rangle, \quad f^{2} \in\left\langle e^{1}, e^{3}\right\rangle, \quad f^{3} \in\left\langle e^{1}, e^{3}, e^{2}\right\rangle .
$$

Proof. We can certainly find an orthonormal basis $\left(f^{i}\right)$ of 1 -forms for which (5.5) is valid, whilst retaining the freedom to act by the stabilizer $U(2)$. Using the latter, we firstly ensure that $f^{1}=k e^{1}$ for some $k \neq 0$, leaving freedom to rotate in the $f^{23}$ plane. Any 2-form in the image of $d$ is divisible by $e^{1}$, so $d\left(f^{14}\right)=-f^{1} \wedge d f^{4}=0$.

Since (5.3) holds in all cases, $f^{23} \wedge e^{13}=0$. This means that we can choose $f^{2}$ to be a linear combination of $e^{1}, e^{3}$. We now have

$$
0=d\left(f^{23}\right)=-f^{2} \wedge d f^{3}=e^{13} \wedge \sigma
$$

for some 1 -form $\sigma \in\left\langle e^{2}, \lambda e^{3}\right\rangle$. But this forces $\sigma \in\left\langle\lambda e^{3}\right\rangle$, and $f^{3}$ cannot have a component in $e^{4}$.

The ingredients are now in place to prove another special case of Theorem 1.1

Theorem 5.2. Let $M$ be a discrete quotient of $N i l^{4}$ admitting a fibration (5.1) over $\mathbb{T}=\mathbb{T}_{z t}^{2}$. Given any invariant almost-Kähler structure $(g, J, \Omega)$ on $M$ and $a$ volume form $\sigma=\mathrm{e}^{F} \Omega^{2}$ with $F \in C^{\infty}(\mathbb{T})$ such that $\int_{\mathbb{T}}\left(\mathrm{e}^{F}-1\right)=0$, the problem (1.2), (1.3) admits a unique solution.

Proof. Let $\left(f^{i}\right)$ be a coframe as in Lemma 5.1. Then we can write

$$
d t=A f^{1}, \quad d z=C f^{1}+B f^{2}
$$

for some $A, C \in \mathbb{R}$ with $A>0$ and $B \neq 0$. We also have

$$
d f^{3}=k f^{12}, \quad d f^{4}=l f^{12}+m f^{13}
$$

with $k, m$ non-zero. Consider now a 1 -form

$$
\alpha=\sum_{i=1}^{4} a_{i} f^{i},
$$

\footnotetext{
${ }^{1} N B$. The inherent filtration would be more memorable had we interchanged $e^{2} \leftrightarrow e^{3}$, but this would have caused bigger notational difficulties elsewhere.
} 
whose coefficients $a_{i}=a_{i}(t, z)$ are functions on the base. We have

$$
\begin{aligned}
d \alpha= & a_{3} k f^{12}+a_{4}\left(l f^{12}+m f^{13}\right)+\sum_{i=1}^{4} d a_{i} \wedge f^{i} \\
= & \left(k a_{3}+l a_{4}+A a_{2, t}-B a_{1, z}\right) f^{12}+\left(m a_{4}+A a_{3, t}+C a_{3, z}\right) f^{13} \\
& \quad+\left(A a_{4, t}+C a_{4, z}\right) f^{14}+B a_{3, z} f^{23}+B a_{4, z} f^{24} .
\end{aligned}
$$

To ensure that $d \alpha$ has type $(1,1)$ relative to $J$, we need

$$
\begin{gathered}
k a_{3}+l a_{4}+A a_{2, t}-B a_{1, z}=0, \\
m a_{4}+A a_{3, t}+C a_{3, z}-B a_{4, z}=0,
\end{gathered}
$$

so that

$$
\Omega+d \alpha=\left(1+A a_{4, t}+C a_{4, z}\right) f^{14}+\left(1+B a_{3, z}\right) f^{23}+B a_{4, z}\left(f^{13}-f^{42}\right)
$$

belongs to $\langle\Omega\rangle+\Lambda^{-}$, as in Theorem 4.4. The volume constraint (1.2) is now

$$
\left(1+A a_{4, t}+C a_{4, z}\right)\left(1+B a_{3, z}\right)-\left(B a_{4, z}\right)^{2}=\mathrm{e}^{F},
$$

where $F=F(t, z)$ is a function on the base.

In view of Proposition 3.2 we need only produce one solution. For this purpose, define

Then

$$
a_{3}=\frac{B}{A} p_{z}-\frac{m}{A} p, \quad a_{4}=p_{t}+\frac{C}{A} p_{z} .
$$

$$
\begin{aligned}
& A\left(m a_{4}+A a_{3, t}+C a_{3, z}-B a_{4, z}\right) \\
& \quad=m\left(A p_{t}+C p_{z}\right)+A\left(B p_{z t}-m p_{t}\right)+C\left(B p_{z z}-m p_{z}\right)-B\left(A p_{z t}+C p_{z z}\right) \\
& \quad=0
\end{aligned}
$$

giving the second equation in (5.8). After a long computation, (5.9) becomes

$$
\left(\frac{B^{2}+C^{2}}{A B^{2}}+p_{t t}-\frac{m C^{2}}{A^{2} B} p_{z}\right)\left(\frac{A}{B^{2}}+p_{z z}-\frac{m}{B} p_{z}\right)-\left(-\frac{C}{B^{2}}+p_{z t}+\frac{m C}{A B} p_{z}\right)^{2}=\frac{1}{B^{2}} \mathrm{e}^{F},
$$

Equations of this type are discussed in the next section. The necessary normalization of $\mathrm{e}^{F}$ is part of our hypothesis, and Theorem 6.2 will guarantee a solution unique up to the addition of a constant.

It remains to satisfy the first equation in (5.8), which becomes

$$
k B p_{z}-m k p+A l p_{t}+l C p_{z}+A^{2} a_{2, t}-A B a_{1, z}=0 .
$$

So far, $a_{1}$ and $a_{2}$ are unconstrained, but they must end up biperiodic. So it suffices to solve the simpler equation

$$
\left(\tilde{a}_{2}\right)_{t}-\left(\tilde{a}_{1}\right)_{z}=q
$$

where $\tilde{a}_{1}, \tilde{a}_{2}$ and $q=-k m p$ are biperiodic, and then add or subtract multiplies of $p$ from $\tilde{a}_{1}, \tilde{a}_{2}$ to get $a_{1}, a_{2}$, respectively. Noting that (5.11) translates into

$$
d\left(\tilde{a}_{1} d t+\tilde{a}_{2} d z\right)=q d t \wedge d z
$$

we first adjust $q$ by a constant so that $\int_{\mathbb{T}} q f^{12}=0$. This means that $[q d t \wedge d z]$ vanishes in $H^{2}(M, \mathbb{R})$, so the 2 -form is exact and (5.11) has a solution with $\tilde{a}_{1}, \tilde{a}_{2} \in$ $C^{\infty}(\mathbb{T})$. 
Just as in Section 4, the solution $d \alpha$ of the Calabi-Yau problem involves a wellidentifiable component on the base as in (4.12), arising from (5.11).

\section{The Generalized Monge-Ampère EQuation}

In this section, we shall work exclusively with functions defined on a 2-torus $\mathbb{T}=\mathbb{T}^{2}$. More precisely, we write $p \in C^{\infty}(\mathbb{T})$ to mean that $p: \mathbb{R}^{2} \rightarrow \mathbb{R}$ is a smooth function satisfying

$$
p(x+1, y)=p(x, y)=p(x, y+1) .
$$

Since we have already dealt with the fibration over $\mathbb{T}_{x y}^{2}$ in Section 4 the pair of coordinates $(x, y)$ in applications will take on the role of either $(t, z)$ (for the previous section) or $(y, t)$ (for the next section).

Given a function $F \in C^{\infty}(\mathbb{T})$, fix a real positive-definite symmetric matrix

$$
\left(\begin{array}{ll}
a & c \\
c & b
\end{array}\right)>0
$$

and two $2 \times 2$ real matrices $\left(l_{i j}\right)$ and $\left(m_{i j}\right)$ satisfying

$$
\begin{aligned}
& m_{11} l_{22}=0, \\
& l_{11} l_{22}-l_{12}^{2}=0, \\
& m_{11} m_{22}-m_{12}^{2}=0, \\
& l_{11} m_{22}+l_{22} m_{11}-2 l_{12} m_{12}=0 .
\end{aligned}
$$

Consider the partial differential equation

$$
\left(a+p_{x x}-l_{11} p_{x}-m_{11} p_{y}\right)\left(b+p_{y y}-l_{22} p_{x}-m_{22} p_{y}\right)-\left(c+p_{x y}-l_{12} p_{x}-m_{12} p_{y}\right)^{2}=\mathrm{e}^{F},
$$

for $p \in C^{\infty}(\mathbb{T})$ satisfying

$$
\left(\begin{array}{cc}
a+p_{x x}-l_{11} p_{x}-m_{11} p_{y} & c+p_{x y}-l_{12} p_{x}-m_{12} p_{y} \\
c+p_{x y}-l_{12} p_{x}-m_{12} p_{y} & b+p_{y y}-l_{22} p_{x}-m_{22} p_{y}
\end{array}\right)>0
$$

at all points on the 2 -torus $\mathbb{T}$.

Example 6.1. After the change of coordinates from $(x, y)$ to $(t, z)$, the equation (5.10) that arose in the previous section is obtained by substituting

$$
\begin{aligned}
a & =\frac{A}{B^{2}}, \quad b=\frac{B^{2}+C^{2}}{A B^{2}}, \quad c=-\frac{C}{B^{2}}, \\
m_{11} & =\frac{m}{B}, \quad m_{22}=\frac{m C^{2}}{A^{2} B}, \quad m_{12}=-\frac{m C}{A B},
\end{aligned}
$$

and $\left(l_{i j}\right)=0$. Conditions 6.2 and 6.4 are verified. The particular case

$$
\begin{aligned}
a & =1, \quad b=1, \quad c=0, \\
m_{11} & =1, \quad m_{22}=m_{12}=0
\end{aligned}
$$

occurs for the standard choice $\Omega=e^{13}+e^{42}$ of symplectic form in Theorem 5.2 . The matrix $\left(l_{i j}\right)$ would intervene if the linear transformation (5.6) were no longer triangular. We have opted for the general form (6.3) so as to emphasize the invariance of our problem under an arbitrary linear change of coordinates on the 2-torus base. 
The main result of this section is

Theorem 6.2. Let $a, b, c,\left(l_{i j}\right),\left(m_{i j}\right)$ and $F$ be as above. Then (6.3) and (6.4) have a solution $p \in C^{2}(\mathbb{T})$ if and only if

$$
\int_{\mathbb{T}}\left(\mathrm{e}^{F}-a b+c^{2}\right)=0 .
$$

Moreover, $p$ is unique modulo addition of a constant, and any $C^{2}$ solution is in $C^{\infty}$.

Proof. We first prove that (6.5) is a necessary condition for (6.3) to have a solution. Using the periodicity of $p$, we see that the following functions integrate to zero over the 2-torus:

$$
\begin{gathered}
p_{x}, \quad p_{y}, \quad p_{x x}, \quad p_{x y}, \quad p_{y y} \\
p_{x x} p_{x}=\frac{1}{2} \partial_{x}\left(p_{x}^{2}\right), \quad p_{y y} p_{y}, \quad p_{x y} p_{x}=\frac{1}{2} \partial_{y}\left(p_{x}^{2}\right), \quad p_{x y} p_{y} .
\end{gathered}
$$

Integrating by parts to obtain the last function, we also have

$$
\int_{\mathbb{T}} p_{y y} p_{x}=-\int_{\mathbb{T}} p_{y} p_{x y}=0, \quad \int_{\mathbb{T}} p_{x x} p_{y}=0 .
$$

If (6.3) has a solution $p \in C^{2}(\mathbb{T})$, then, from above and assumptions on $\left(l_{i j}\right),\left(m_{i j}\right)$,

$$
\begin{aligned}
\int_{\mathbb{T}} \mathrm{e}^{F}= & \int_{\mathbb{T}}\left[\left(a+p_{x x}\right)\left(b+p_{y y}\right)-\left(c+p_{x y}\right)^{2}\right. \\
& \left.+\left(l_{11} p_{x}+m_{11} p_{y}\right)\left(l_{22} p_{x}+m_{22} p_{y}\right)-\left(l_{12} p_{x}+m_{12} p_{y}\right)^{2}\right] \\
= & \left(a b-c^{2}\right)|\mathbb{T}|+\int_{\mathbb{T}}\left[p_{y y} p_{x x}-p_{x y}^{2}\right] \\
& +\int_{\mathbb{T}}\left[p_{x}^{2}\left(l_{11} l_{22}-l_{12}^{2}\right)+p_{y}^{2}\left(m_{11} m_{22}-m_{12}\right)\right. \\
& \left.+p_{x} p_{y}\left(l_{11} m_{22}+l_{22} m_{11}-2 l_{12} m_{12}\right)\right] \\
= & \left(a b-c^{2}\right)|\mathbb{T}|+\int_{\mathbb{T}}\left[p_{y y} p_{x x}-p_{x y}^{2}\right] .
\end{aligned}
$$

Similarly,

$$
\int_{\mathbb{T}}\left[p_{y y} p_{x x}-p_{x y}^{2}\right]=\int_{\mathbb{T}}\left[-p_{y} p_{x x y}-p_{x y}^{2}\right]=0 .
$$

It follows that (6.5) holds.

Now we prove the uniqueness of solutions modulo addition of constants. Let $p$ and $\widetilde{p}$ be two solutions of (6.3) and (6.4). Let $h$ be a constant such that

$$
\min _{\mathbb{T}}[(p+h)-\tilde{p}]=0 \text {. }
$$

Clearly, $p+h$ is also a solution of (6.3) and (6.4). By the mean value theorem, we have

$$
\begin{aligned}
0= & a_{11}(x, y) \partial_{y y}[(p+h)-\tilde{p}]+2 a_{12}(x, y) \partial_{x y}[(p+h)-\tilde{p}] \\
& +a_{22}(x, y) \partial_{y y}[(p+h)-\tilde{p}]+b(x, y) \partial_{y}[(p+h)-\tilde{p}]
\end{aligned}
$$


where $\left(a_{i j}(x, y)\right)$ is a positive-definite symmetric matrix function which, like $b(x, y)$, is continuous on $\mathbb{T}$. Thus, by the strong maximum principle, $(p+h)-\widetilde{p} \equiv 0$. The uniqueness result follows.

Since the equation is elliptic and all data is smooth, a $C^{2}$ solution $p$ is in $C^{\infty}$.

To prove the existence part of the theorem, we use the method of continuity. This requires a priori estimates which we derive below.

Lemma 6.3. Let $f \in C^{2}(\mathbb{R})$ be a 1-periodic function satisfying, for some $\alpha, \beta \in \mathbb{R}$,

$$
f^{\prime \prime}(s)+\alpha f^{\prime}(s) \geqslant \beta, \quad-\infty<s<\infty .
$$

Then

$$
\left|f^{\prime}(s)\right| \leqslant 2|\beta| \mathrm{e}^{2|\alpha|}, \quad \forall s \in \mathbb{R} \text {. }
$$

Proof. We know that

$$
\frac{d}{d s}\left[\mathrm{e}^{\alpha s} f^{\prime}(s)\right] \geqslant \beta \mathrm{e}^{\alpha s}
$$

Since $f$ is 1 -periodic, there exists $\bar{s} \in[0,1)$ such that $f^{\prime}(\bar{s})=0$. It follows that

$$
\mathrm{e}^{\alpha s} f^{\prime}(s) \geqslant \beta \int_{\bar{s}}^{s} \mathrm{e}^{\alpha t} d t, \quad s \geqslant 1 .
$$

This implies

$$
f^{\prime}(s) \geqslant \mathrm{e}^{-\alpha s} \beta \int_{\bar{s}}^{s} \mathrm{e}^{\alpha t} d t \geqslant-2|\beta| \mathrm{e}^{2|\alpha|}, \quad 1 \leqslant s \leqslant 2 .
$$

Similarly,

and

$$
-\mathrm{e}^{\alpha s} f^{\prime}(s) \geqslant \beta \int_{s}^{\bar{s}} \mathrm{e}^{\alpha t} d t, \quad s \leqslant 0,
$$

$$
-f^{\prime}(s) \geqslant \beta \mathrm{e}^{-\alpha s} \int_{s}^{\bar{s}} \mathrm{e}^{\alpha t} d t \geqslant-2|\beta| \mathrm{e}^{2|\alpha|}, \quad-1 \leqslant s \leqslant 0 .
$$

The desired estimate follows from the above and the 1-periodicity of $f$.

For $0 \leqslant t \leqslant 1$, consider the equation

$$
\begin{aligned}
& \left(a+p_{x x}-t l_{11} p_{x}-t m_{11} p_{y}\right)\left(b+p_{y y}-t l_{22} p_{x}-t m_{22} p_{y}\right) \\
& -\left(c+p_{x y}-t l_{12} p_{x}-t m_{12} p_{y}\right)^{2}=t \mathrm{e}^{F}+(1-t)\left(a b-c^{2}\right)
\end{aligned}
$$

together with the condition

$$
\left(\begin{array}{ll}
a+p_{x x}-t l_{11} p_{x}-t m_{11} p_{y} & c+p_{x y}-t l_{12} p_{x}-t m_{12} p_{y} \\
c+p_{x y}-t l_{12} p_{x}-t m_{12} p_{y} & b+p_{y y}-t l_{22} p_{x}-t m_{22} p_{y}
\end{array}\right)>0 \quad \text { on } \quad \mathbb{T} .
$$

For this problem, we have

Lemma 6.4. For $0 \leqslant t \leqslant 1$, let $p \in C^{2}(\mathbb{T})$ be a solution of (6.7) and (6.8). Then

$$
|\nabla p| \leqslant C \quad \text { on } \quad \mathbb{T},
$$

and

$$
\left|p-\frac{1}{|\mathbb{T}|} \int_{\mathbb{T}} p\right| \leqslant C \quad \text { on } \quad \mathbb{T},
$$

where $C$ depends only on $F$ and $a, b, c,\left(l_{i j}\right),\left(m_{i j}\right)$. 
Proof. We know that $m_{11} l_{22}=0$. If $l_{22} \neq 0$, then $m_{11}=0$, and, in view of (6.8), $a+p_{x x}-t l_{11} p_{x}$ and $b+p_{y y}-t l_{22} p_{x}-t m_{22} p_{y}$ are positive on $\mathbb{T}$. Applying Lemma 6.3 with $(\alpha, \beta)$ equal to $\left(-t l_{11},-a\right)$ leads to $\left|p_{x}\right| \leqslant C$. Now we apply Lemma 6.3 with

$$
(\alpha, \beta)=\left(-t m_{22},-b-\left\|l_{22} p_{x}\right\|_{L^{\infty}}\right)
$$

to obtain $\left|p_{y}\right| \leqslant C$. If $m_{11} \neq 0$, then $l_{22}=0$, and we obtain (6.9) similarly. Estimate (6.10) follows from (6.9), bearing in mind the definition (6.1) of $\mathbb{T}$.

Lemma 6.5. For $0 \leqslant t \leqslant 1$, let $p \in C^{2}(\mathbb{T})$ be a solution of (6.7) and (6.8). Then

$$
\left|\nabla^{2} p\right| \leqslant C \quad \text { on } \mathbb{T},
$$

where $C$ depends only on $F$ and $a, b, c,\left(l_{i j}\right),\left(m_{i j}\right)$.

Proof. With Lemma 6.4, this follows from [21, Theorem 1].

Lemma 6.6. For $0 \leqslant t \leqslant 1$, let $p \in C^{2}(\mathbb{T})$ be a solution of 6.7 and (6.8). Then $p \in C^{\infty}(\mathbb{T})$ and for any positive integer $k$,

$$
\left\|p-\frac{1}{|\mathbb{T}|} \int_{\mathbb{T}} p\right\|_{C^{k}(\mathbb{T})} \leqslant C,
$$

where $C$ depends only on $k, F$, and $a, b, c,\left(l_{i j}\right),\left(m_{i j}\right)$.

Proof. With Lemmas 6.4 and 6.5, it follows from a theorem of Nirenberg [17] that for some constant $0<\mu<1$, and some constant $C$,

$$
\left\|p-\frac{1}{|\mathbb{T}|} \int_{\mathbb{T}} p\right\|_{C^{2, \mu}(\mathbb{T})} \leqslant C
$$

for all solutions of (6.7) and (6.8), and for all $0 \leqslant t \leqslant 1$. The higher derivative estimates then follow from Schauder estimates.

For $0<\mu<1$ and $0 \leqslant t \leqslant 1$, let

$$
\begin{aligned}
X^{2, \mu} & :=\left\{p \in C^{2, \mu}(\mathbb{T}) \mid \int_{\mathbb{T}} p=0\right\}, \\
X^{\mu} & :=\left\{q \in C^{\mu}(\mathbb{T}) \mid \int_{\mathbb{T}} q=0\right\},
\end{aligned}
$$

and

$$
\begin{aligned}
S_{t}(p):=\left(a+p_{x x}-t\right. & \left.l_{11} p_{x}-t m_{11} p_{y}\right)\left(b+p_{y y}-t l_{22} p_{x}-t m_{22} p_{y}\right) \\
& -\left(c+p_{x y}-t l_{12} p_{x}-t m_{12} p_{y}\right)^{2}-\left[t \mathrm{e}^{F}+(1-t)\left(a b-c^{2}\right)\right] .
\end{aligned}
$$

Integration by parts $(\sqrt{6.6})$ and the equations preceding it) implies that

$$
S_{t}: X^{2, \mu} \rightarrow X^{\mu} \text {. }
$$

Clearly, for any $p \in X^{2, \mu}$,

$$
S_{t}^{\prime}(p): X^{2, \mu} \rightarrow X^{\mu}
$$

Moreover, by elliptic theories, $S_{t}^{\prime}(p)$ is an isomorphism from $X^{2, \mu}$ to $X^{\mu}$ for any $p \in X^{2, \mu}$ satisfying (6.8).

At $t=0$, the zero function $p \equiv 0$ is a solution of (6.7). Since the linearized operator $S_{t}^{\prime}(p)$ is an isomorphism from $X^{2, \mu}$ to $X^{\mu}$, and since we have established a priori estimates in Lemma 6.6, the solvability follows from the standard method of continuity. In this way, Theorem 6.2 is established. 


\section{The 2-STEP CASE REVISITED}

We return to the study of case $\left(1_{\mathrm{a}}\right)$ and a discrete quotient $M=\Gamma_{0} \backslash\left(N i l^{3} \times S^{1}\right)$, with the invariant coframe (4.1) that we reproduce here for convenience:

$$
e^{1}=d y, \quad e^{2}=d x, \quad e^{3}=d t, \quad e^{4}=d z-x d y .
$$

Consider the fibration

$$
\pi_{y t}: M \longrightarrow \mathbb{T}_{y t}^{2},
$$

where a volume form on the base is $e^{13}$.

As we remarked in Section 3 , the fibres may or may not be Lagrangian, depending on the choice of symplectic form. For example, whilst $e^{14}+e^{23}$ restricts to zero, $e^{13}+e^{42}$ is non-degenerate on the fibres of $\pi_{y t}$. We are unable to say much about the situation of a symplectic fibration without the hypothesis on $J$ that was used in Proposition 3.1. Next, we turn out attention to the Lagrangian case.

Apply Lemma 5.1 to retrieve a coframe $\left(f^{i}\right)$ respecting the filtration determined by the ordered basis $\left(e^{1}, e^{3}, e^{2}, e^{4}\right)$, and write

$$
d f^{4}=l f^{12}+m f^{13}, \quad m \neq 0 .
$$

This corresponds to (5.7), except that $k=0$ in the present context. One may easily repeat the calculations in the proof of Theorem 5.2 with $k$ (that played little role) zero. We obtain the same generalized Monge-Ampère equation

$$
\left(\frac{B^{2}+C^{2}}{A B^{2}}+p_{y y}-\frac{m C^{2}}{A^{2} B} p_{t}\right)\left(\frac{A}{B^{2}}+p_{t t}-\frac{m}{B} p_{t}\right)-\left(-\frac{C}{B^{2}}+p_{y t}+\frac{m C}{A B} p_{t}\right)^{2}=\frac{1}{B^{2}} \mathrm{e}^{F},
$$

that can be solved by the techniques of Section 6 . In this case the final step reduces to

$$
A l p_{y}+l C p_{t}+A^{2} a_{2, y}-A B a_{1, t}=0,
$$

which can be simply solved by setting

$$
a_{1}=\frac{l C}{A B} p, \quad a_{2}=-\frac{l}{A} p
$$

In conclusion,

Corollary 7.1. Let $(g, J, \Omega)$ be an invariant almost-Kähler structure on $M$ for which $\Omega$ restricts to zero on the fibres of $\pi_{y t}$ over $\mathbb{T}=\mathbb{T}_{y t}^{2}$. Let $\sigma=\mathrm{e}^{F} \Omega^{2}$ be a volume form with $F \in C^{\infty}(\mathbb{T})$ and $\int_{\mathbb{T}}\left(\mathrm{e}^{F}-1\right)=0$. Then the associated Calabi-Yau problem has a unique solution.

7.1. Manifolds belonging to $\left(1_{\mathrm{b}}\right)$ and $\left(1_{\mathrm{a}}\right)$. Consider now a $T^{2}$ fibration

$$
\pi: M=\Gamma \backslash X \rightarrow \mathbb{T}^{2},
$$

where $X=N i l^{3} \times \mathbb{R}$ and the generators of $\Gamma$ are given by either $\left(1_{\mathrm{b}}\right)$ or $\left(1_{\mathrm{c}}\right)$. In this case, $\Gamma$ is not a lattice of $X$, but it contains a lattice $\Gamma_{0}$ of $X$ such that $\Gamma_{0} \backslash \Gamma=\mathbb{Z}_{2}$. Therefore there exists a covering map $p: \Gamma_{0} \backslash X \rightarrow \Gamma \backslash X$ which preserves the $T^{2}$ bundle structure over $\mathbb{T}^{2}$. An almost-Kähler structure in this context is called invariant if it is left-invariant on $X$ and invariant by $\Gamma$. In particular every invariant almost-Kähler structure on $\Gamma \backslash X$ induces an invariant almost-Kähler structure on $\Gamma_{0} \backslash X$. Since $p$ preserves the $T^{2}$-bundle, Corollary 7.1 implies that the Calabi-Yau problem for $T^{2}$-invariant volume form can be solved even in these two cases. 
7.2. Representation theory. The fibrations $\pi_{x y}, \pi_{z t}$ of the Kodaira-Thurston manifold are intimately related to its function theory and unitary representations of the Heisenberg group $\mathrm{Nil}^{3}$. We describe this briefly.

For simplicity, ignore the $\mathbb{R}$ factor and work on the nilmanifold $M^{3}=\mathbb{Z}^{3} \backslash N i l^{3}$, where $\mathbb{Z}^{3}$ is the standard integer lattice (see (2.1) $)$. Consider the action of $N_{i l}^{3}$ on $f \in L^{2}(\mathbb{R})$ defined by setting

$$
\left(H_{x, y, z} \cdot f\right)(u)=\mathrm{e}^{2 \pi i k(z+y u)} f(x+u),
$$

where $H_{x, y, z}$ is the matrix (2.1) and (here) $k$ is an integer. This makes $L^{2}(\mathbb{R})$ into a unitary representation of $N i l^{3}$ that we denote by $V_{k}$.

Following the discussion on [14, page 6], for any smooth function $f \in L^{2}(\mathbb{R})$, we set

$$
F(x, y, z)=\sum_{n \in \mathbb{Z}}\left(H_{x, y, z} \cdot f\right)(n)=\mathrm{e}^{2 \pi i k z} \sum_{n \in \mathbb{Z}} f(x+n) \mathrm{e}^{2 \pi i n k y} .
$$

By its construction, $F=F_{f}$ is a well-defined function $M^{3} \rightarrow \mathbb{C}$. Indeed

$$
F(x+a, y+b, z+c+a y)=F(x, y, z), \quad a, b, c \in \mathbb{Z},
$$

using the coordinates of (2.1). In particular, one may regard $x, y$ as defined on $\mathbb{T}_{x y}^{2}$ and $y$ as defined on $\mathbb{T}_{y t}^{2}$, but we are now considering functions outside the $T^{2}$ invariant class that we have previously considered. If we take $f(x)=\mathrm{e}^{-\pi x^{2}}$, then

$$
F(x, y, z)=e^{2 \pi i k z} \vartheta(k y+i x) f(x),
$$

where $\vartheta$ is a classical theta function.

The mapping $f \mapsto F$ given by (7.1) realizes $V_{k}$ as a summand of $L^{2}\left(M^{3}\right)$. A more subtle discussion leads to a Peter-Weyl type decomposition

$$
L^{2}\left(M^{3}\right) \cong L^{2}\left(\mathbb{T}^{2}\right) \oplus \bigoplus_{k \in \mathbb{Z}}|k| V_{k},
$$

in which $V_{k}$ occurs as an isotypic component of multiplicity $|k|$ (see $[3$ and references therein). In our context, one might first hope to extend the class of assigned volume forms to functions on $M^{3}$, and analyse their behaviour under the relevant differential operators with the aid of (7.3). Generalizations of the latter hold for $L^{2}$ spaces of sections of holomorphic line bundles over the Kodaira-Thurston manifold [6, 14].

\section{Almost-Kähler Structures FOr $S o l^{3} \times \mathbb{R}$}

In this final section, we make some observations regarding Calabi-Yau problems for the manifolds belonging to the families $\left(3_{\mathrm{a}}\right)$ and $\left(3_{\mathrm{b}}\right)$.

As in Section 7 we assume that $M$ is a solvmanifold (i.e. that $\Gamma$ is a lattice), and then we use the observation of subsection 7.1 to generalize to the case of infrasolvmanifolds. Let

$$
\pi: M=\Gamma \backslash \mathbb{R}^{4} \longrightarrow \mathbb{T}_{z t}^{2}
$$

be the relevant $T^{2}$-bundle with $\pi(x, y, z, t)=(z, t)$ and $\Gamma$ a lattice of $G$. In the notation of Section 2, we begin with the coframe

$$
e^{1}=d t, \quad e^{2}=d z, \quad e^{3}=\mathrm{e}^{t} d x, \quad e^{4}=\mathrm{e}^{-t} d y .
$$

Each tangent space to the fibres of $\pi_{z t}$ is represented by the annihilator $\left\langle e^{1}, e^{2}\right\rangle^{\circ}$, and $e^{12}$ is a volume form on the base $\mathbb{T}_{z t}^{2}$. 
A special feature of the geometry modelled on (8.1) is that the transversal subspace $\left\langle e^{3}, e^{4}\right\rangle^{\circ}$ is tangent to a distribution $D$ that is also integrable. This is an immediate consequence of the fact that the invariant forms $e^{3}, e^{4}$ generate a differential ideal. Moreover,

Lemma 8.1. Let $(g, J, \Omega)$ be any invariant almost-Kähler structure on $M$. The distribution $D$ is J-holomorphic, and there exists an orthonormal basis $\left(f^{i}\right)$ such that

$$
\Omega=f^{12}+f^{34}
$$

and

$$
f^{1} \in\left\langle e^{1}\right\rangle, \quad f^{3} \in\left\langle e^{3}\right\rangle, \quad f^{4} \in\left\langle e^{3}, e^{4}\right\rangle .
$$

Proof. Let $\left(f^{i}\right)$ be an orthonormal basis of 1-forms for which (8.2) is valid and $f^{1} \in\left\langle e^{1}\right\rangle$. Since any 2-form in the image of $d$ is divisible by $e^{1}$, we get $d\left(f^{12}\right)=$ $-f^{1} \wedge d f^{2}=0$. Thus,

$$
0=d\left(f^{34}\right)=-f^{3} \wedge d f^{4}+d f^{3} \wedge f^{4}=e^{1} \wedge\left(f^{3} \wedge \sigma+f^{4} \wedge \tau\right),
$$

for some 1-forms $\sigma, \tau \in\left\langle e^{3}, e^{4}\right\rangle \cap\left\langle f^{3}, f^{4}\right\rangle$. If $\sigma \wedge \tau=0$, then $\left\langle f^{3}, f^{4}\right\rangle$ contains a closed 1-form that cannot be proportional to $e^{1}$. Then then $d\left(f^{34}\right) \neq 0$. Therefore $\sigma, \tau$ are linearly independent and $\left\langle f^{3}, f^{4}\right\rangle=\left\langle e^{3}, e^{4}\right\rangle$. Finally, we may rotate in this plane so as to select $f^{3} \in\left\langle e^{3}\right\rangle$.

It now follows that $f^{4}=J f^{3}$ and so $D$ is $J$-invariant.

If $f^{2} \in\left\langle e^{1}, e^{2}\right\rangle$, then $\pi_{z t}$ is also $J$-holomorphic, and we can apply Proposition 3.1 to obtain an elementary solution:

Corollary 8.2. Let $(g, J, \Omega)$ be an invariant almost-Kähler structure on $M$ for which $\pi_{z t}$ is J-holomorphic, and let $\sigma=\mathrm{e}^{F} \Omega$ be a normalized volume form with $\mathrm{e}^{F} \in C^{\infty}\left(\mathbb{T}_{z t}^{2}\right)$. Then the Calabi-Yau equation $(\Omega+d \alpha)^{2}=\sigma$ has a unique solution.

One can apply the same argument for a volume form that is constant along the leaves of the associated foliation:

Corollary 8.3. Let $(g, J, \Omega)$ be an invariant almost-Kähler structure on $M$, and let $\sigma=\mathrm{e}^{F} \Omega$ be a normalized volume form such that $d F \wedge e^{34}=0$. Then the Calabi-Yau equation $(\Omega+d \alpha)^{2}=\sigma$ has a unique solution.

Proof. Let $\left(f^{i}\right)$ a coframe of 1-forms as in Lemma 8.1. A solution of the Calabi-Yau equation is simply given by $\tilde{\Omega}=f^{12}+\mathrm{e}^{F} f^{34}$. Indeed, $\tilde{\Omega}$ is compatible with respect to $J$ since $J$ preserves $f^{34}$, and $\tilde{\Omega}-\Omega=\left(\mathrm{e}^{F}-1\right) f^{34}$ is exact since the normalization

$$
\int_{M}\left(\mathrm{e}^{F}-1\right) f^{1234}=0
$$

implies that it has zero cup product with $H^{2}(M, \mathbb{R})=\left\langle\left[f^{12}\right],\left[f^{34}\right]\right\rangle=\left\langle\left[e^{12}\right],\left[e^{34}\right]\right\rangle$.

It is interesting to compare the dual situations highlighted by the last two results. In Corollary 8.2 , the choice of $\Omega$ (or, alternatively, $J$ ) is restricted but $F$ is a free function on the base. On the other hand, Corollary 8.3 refers to an arbitrary invariant almost-Kähler structure, though $\Omega$ and $J$ are in practice already constrained by the geometry. Because of the nature of the foliation one is dealing with, the applicable class of functions is restricted, as our final example illustrates. 
Example 8.4. This is a sequel to Example 2.1 in which $M=M^{3} \times S^{1}$, with $z$ a coordinate on $S^{1}$. The leaves of $D$ have the form $C \times S^{1}$, where $C$ is an integral curve of the form

$$
(x, y, t)=\left(x_{0} / \sigma^{k}, y_{0} \sigma^{k}, t\right), \quad k \delta \leqslant t<(k+1) \delta .
$$

For generic $\left(x_{0}, y_{0}\right)$ this curve is dense in $M^{3}$, and so any smooth function constant on $C$ is constant on $M^{3}$. Therefore the hypothesis $d F \wedge e^{34}=0$ implies that $F \in C^{\infty}\left(S^{1}\right)$, and the normalization is $\int_{S^{1}} F(z) d z=0$.

8.1. Conclusion. Earlier in the paper, we successfully solved the Calabi-Yau problem for volume forms invariant by a 2-torus action, relative to various fixed almostKähler structures and Lagrangian fibrations to a 2-torus base $\mathbb{T}^{2}$. The $T^{2}$-invariance is a natural hypothesis, in view of the analogy with toric geometry in which moment mappings play the role of the fibrations.

On the other hand, the examples in the present section show that, in a more general context, it is futile to restrict the class of volume forms. The manifold $\Gamma \backslash S o l^{3}$ exhibits geometry of a very different type, involving an action by the $2 \times 2$ diagonal matrix $\varphi(t)$ (see Section 2) also seen on the unit tangent bundle $P S L(2, \mathbb{Z}) \backslash P S L(2, \mathbb{R})$ of the modular surface $[10$.

The theory described in subsection 7.2 , or rather its generalizations to four dimensions tailored to symplectic geometry [6, 14, is likely to be relevant in solving the problem in a general non-Lagrangian setting.

In another direction, one can vary the ambient almost-Kähler structure. As a first step, the result of Corollary 4.5 can be extended to "separable" symplectic forms such as $\Omega(x, y)=f(x) e^{14}+g(y) e^{23}$. Such calculations lead one to postulate further Calabi-Yau existence results.

\section{ACKNOWLEDGMENTS}

The authors thank Pawel Nurowski for a crucial observation that led to the reduction accomplished in Section 4, and Valentino Tosatti for his encouragement. Aspects of the work were described at the Workshop on Kähler and related geometries (Nantes 2009) and XIX International Fall Workshop on Geometry and Physics (Porto 2010).

\section{REFERENCES}

[1] E. Abbena, An example of an almost Kähler manifold which is not Kählerian, Boll. Un. Mat. Ital. (6) 3-A (1984), no. 3, 383-392. MR769169 (86a:53036)

[2] M. Abreu, R. Sena-Dias, Scalar-flat Kähler metrics on non-compact symplectic toric 4manifolds, arXiv:0910.5466

[3] L. Auslander, J. Brezin, Translation-invariant subspaces in $L^{2}$ of a compact nilmanifold. I, Invent. Math. 20 (1973), 1-14. MR0322100(48:464)

[4] C. Bock, On low-dimensional solvmanifolds, arXiv:0903.2926

[5] S. K. Donaldson, Two-forms on four-manifolds and elliptic equations. Inspired by S. S. Chern, 153-172, Nankai Tracts Math. 11, World Scientific, Hackensack NJ, 2006. MR 2313334 (2008c:53090)

[6] D. V. Egorov, Theta functions on $T^{2}$-bundles over $T^{2}$ with the zero Euler class, Siberian Math. J. 50 (2009), no. 4, 647-657. MR2583619(2011c:32037)

[7] R. O. Filipkiewicz, Four-dimensional geometries, Ph.D. thesis, University of Warwick, 1984.

[8] A. Fino, H. Pedersen, Y.-S. Poon, M. W. Sørensen, Neutral Calabi-Yau structures on Kodaira manifolds, Comm. Math. Phys. 248 (2004), no. 2, 255-268. MR2073135 (2005d:32044)

[9] H. Geiges, Symplectic structures on $T^{2}$-bundles over $T^{2}$, Duke Math. J. 67 (1992), 539-555. MR $1181312(93 \mathrm{i}: 57036)$ 
[10] E. Ghys, Knots and dynamics. International Congress of Mathematicians. Vol I, 247-277, European Math. Soc., Zürich, 2007. MR2334193 (2008k:37001)

[11] J. A. Hillman, Four-manifolds, geometries and knots, Geometry \& Topology Monographs 5. Mathematical Sciences Publishers, Berkeley CA, 2002. MR.1943724 (2003m:57047)

[12] N. J. Hitchin, The moduli space of special Lagrangian sub-manifolds, Annali Scuola Norm. Sup. Pisa 25 (1997), 503-515. MR.1655530 (2000c:32075)

[13] G. Ketsetzis, S. Salamon, Complex structures on the Iwasawa manifold, Adv. Geom. 4 (2004), no. 2, 165-179. MR2055676 (2005g:22008)

[14] W. D. Kirwin, A. Uribe, Theta functions on the Kodaira-Thurston manifold, Trans. Amer. Math. Soc. 362 (2010), no. 2, 897-932. MR2551510(2011d:53228)

[15] K. Kodaira, On the structure of compact complex analytic surfaces, I, Amer. J. Math. 86 (1964), 751-798. MR0187255 (32:4708)

[16] Y. Y. Li, Some existence results of fully nonlinear elliptic equations of Monge-Ampère type, Comm. Pure Appl. Math. 43 (1990), 233-271. MR1038143 (91b:58269)

[17] L. Nirenberg, On nonlinear elliptic partial differential equations and Hölder continuity, Comm. Pure Appl. Math. 6 (1953), 103-156. MR0064986 (16:367c)

[18] Y. S. Poon, Extended deformation of Kodaira surfaces, J. Reine Angew. Math. 590 (2006), 45-65. MR2208128 (2007e:32009)

[19] K. Sakamoto, S. Fukuhara, Classification of $T^{2}$-bundles over $T^{2}$, Tokyo J. Math. 6 (1983), 311-327. MR732086 (85h:55021)

[20] S. M. Salamon, Complex structures on nilpotent Lie algebras, J. Pure Appl. Algebra 157 (2001), no. 2-3, 311-333. MR1812058 (2002g:53089)

[21] F. Schulz, Second-derivative estimates for solutions of two-dimensional Monge-Ampère equations, Proc. Amer. Math. Soc. 111 (1991), 101-110. MR1031671 (91e:35038)

[22] W. P. Thurston, Some simple examples of symplectic manifolds, Proc. Amer. Math. Soc. 55 (1976), 467-468. MR0402764 (53:6578)

[23] V. Tosatti, B. Weinkove, S. T. Yau, Taming symplectic forms and the Calabi-Yau equation, Proc. London Math. Soc. 97 (2008), no. 2, 401-424. MR2439667 (2009h:32032)

[24] V. Tosatti, B. Weinkove, Estimates for the complex Monge-Ampère equation on Hermitian and balanced manifolds, Asian J. Math. 14 (2010), 19-40. MR2726593 (2011h:32043)

[25] V. Tosatti, B. Weinkove, The Calabi-Yau equation on the Kodaira-Thurston manifold, $J$. Inst. Math. Jussieu 10 (2011), no. 2, 437-447. MR2787695

[26] M. Ue, On the 4-dimensional Seifert fiberings with euclidean space orbifolds, in $A$ fête of topology, 471-523, Academic Press, Boston, 1988. MR928414 (89c:55015)

[27] M. Ue, Geometric 4-manifolds in the sense of Thurston and Seifert 4-manifolds I, J. Math. Soc. Japan 42 (1990), no. 3, 511-540. MR1056834 (91k:57023)

[28] S. T. Yau, On the Ricci curvature of a compact Kähler manifold and the complex MongeAmpère equation, I, Comm. Pure Appl. Math. 31 (1978), no. 3, 339-411. MR480350 (81d:53045)

Dipartimento di Matematica, Università di Torino, Via Carlo Alberto 10, 10123 TORINO, ITALIA

E-mail address: annamaria.fino@unito.it

Department of Mathematics, Rutgers University, 110 Frelinghuysen Road, PiscatAWAY, NEW JERSEY 08854

E-mail address: yyli@math.rutgers.edu

Dipartimento di Matematica, Politecnico di Torino, Corso Duca degli Abruzzi 24, 10129 Torino, Italia - And - Department of Mathematics, King's College London, Strand, LONDON, WC2R 2LS, United Kingdom

E-mail address: simon.salamon@kcl.ac.uk

Dipartimento di Matematica, Università di Torino, Via Carlo Alberto 10, 10123 TORINO, ITALIA

E-mail address: luigi.vezzoni@unito.it 